\title{
EFFECTS OF $\gamma$-AMINOBUTYRIC ACID (GABA), $\gamma$-AMINOBUTYRYL CHOLINE (GABA-CH) AND THEIR RELATED SUBSTANCES ON THE CORTICAL ACTIVITY
}

\author{
HIDEHIKO TAKAHASHI, AKIRA NAGASHIMA, CHIKAO KOSHINO \\ AND HISASHI TAKAHASHI*
}

Department of Physiology, Tokyo Medical College, Tokyo

In 1952 Takahashi and Tsukada (1) reported a preliminary investigation about the actions of $\omega$-aminoacids on the cortical activity, but at that time they could not come to any apparent conclusion about the action of $r$-aminobutyric acid. In recent years the inhibitory action of this acid on the electrical activity of the cortex has been noticed by some investigators $(2,3,4)$.

In this study we have reexamined the action of $r$-aminobutyric acid and investigated the effects of $r$-aminobutyryl choline and other related substances on the cortical activity.

\section{METHODS}

Experiments were performed on 70 rabbits and a few cats. The convulsive action of drugs was as a rule observed in unanesthetized animals, but evoked potentials were recorded from phenobarbital- or urethane-anesthetized ones.

For recordings of the electrical activites of the cortex, bipolar or unipolar silver wire electrodes with a ball tip of about $0.5 \mathrm{~mm}$ in diameter were placed on the pial surface.

Evoked potentials were recorded by means of condenser-coupled amplifier (time constant $0.5 \mathrm{sec}$ ) led to a dual beam oscilloscope. EEG was recorded by a two- channel ink-writing apparatus. For electrical stimulation, square pulses of $0.02-0.1 \mathrm{msec}$ were delivered from an isolation unit. Stimulating electrodes were of the same type as surface-leading ones.

Topical applications of chemicals were performed with squares of filter papers approximately $2 \times 2 \mathrm{~mm}$. Among the chemicals used by us, $\beta$-alanine and $\varepsilon$-aminocaproic acid (EACA, m.p. $203^{\circ} \mathrm{C}$ ) were offered by Mitsubishi Kasei Laboratory. $\gamma$-Aminobutyric acid (GABA, m.p. $199-201^{\circ} \mathrm{C}$ ), $\gamma$-aminobutyryl choline chloride hydrochloride (GABA-Ch, m.p. $193^{\circ} \mathrm{C}$ ), glutamyl choline and carbamyl choline chloride were given by the Scientific Department of Daiich Seiyaku Co. Metrazol (m.p. $57-60^{\circ} \mathrm{C}$ ) was offered by Sankyo Seiyaku Co. Other chemicals were of commercial supply. The purity of GABA was especially cautiously checked. Only a slight impurity of GABA caused striking changes in its biological action.

Received for publication February 7, 1959.

* 高橋日出彦, 長島 璋, 腰野千賀雄, 高橋 長 
RESULTS

\section{The action of some amino acids on the rabbit's cerebral cortex.}

The action of $\omega$-aminoacids on the electrical activity of the cortex was previously observed by Purpura et al. (2) in the cat. We observed the action of $\omega$-aminoacids and some other aminoacids on the spontaneous electrical activity of the cortex in rabbits.

In concentrations from $10^{-3}$ to $1 \mathrm{~mol} / 1$, glycine, $\beta$-alanine, GABA and $\delta$-aminovaleric acid did not show any excitatory effect on the spontaneous electrical activity, when applied topically, but $\varepsilon$-aminocaproic acid (EACA) caused firing of cortical elements at $6 \times 10^{-1} \mathrm{~mol} / 1$. Among the substances above described, $\beta$-alanine and GABA inhibited EACA-discharge. GABA inhibited completely the convulsive action of $10 \%$ EACA in the concentration of $1-2 \times 10^{-1} \mathrm{~mol} / 1$ and $\beta$-alanine at $1.3 \mathrm{~mol} / 1$. $\delta$-Aminovaleric acid (Fluka Co.) did not show any effect at the concentration of $1 \mathrm{~mol} / 1$.

In fig. 1 the relative excitatory or inhibitory powers of $\omega$-aminoacids are plotted as a function of the distance between carboxyl- and amino-group. The inhibitory action of GABA on the EACA-excitation is estimated arbitrarily at 10 .

In fig. 2 the mode of action of GABA on EACA-spikes is shown by a typical experiment. $10 \%$ EACA caused rhythmical firings at some constant intervals which consisted of a group of surface negative waves. These discharges did not disappear after washing with fresh Ringer solution. Then, $1.0 \times 10^{-1} \mathrm{~mol} / 1 \mathrm{GABA}$ was applied to the same locus. Reversal in polarity of spikes occurred at once. In this period, surface positive spikes continued rather longer. At length, spike discharge disappeared completely.

Among the isomers of aminobutyric acid, both $\alpha$-amino- $n$-butyric acid and $\alpha$ aminoisobutyric acid at $1 \mathrm{~mol} / \mathrm{l}$ showed neither inhibitory nor facilitatory action on the spontaneous electrical activity of the cortex. About the action of $\alpha$-amino$n$-butyric acid, Purpura et al. (2) had already obtained similar results in the cat's cortex. Whereas, $\beta$-aminoisobutyric acid in the concentrations of $0.1-1 \mathrm{~mol} / 1$ were found to elicit surface positive waves (fig. 3). And, it showed an inhibitory action on EACA-spike. The findings described above suggest that in the inhibitory action

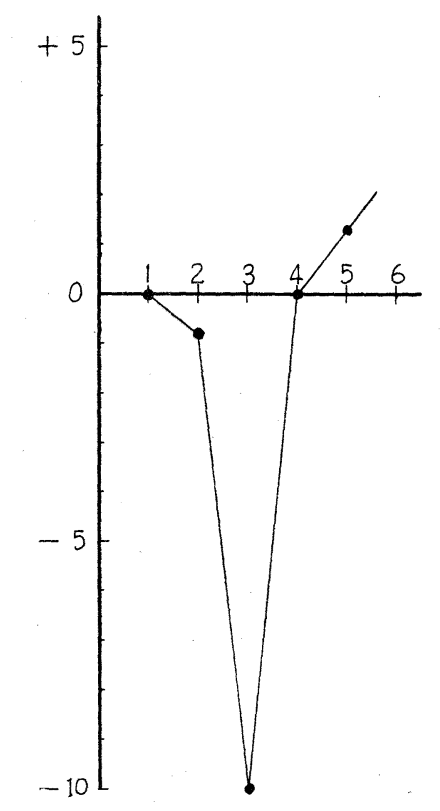

FIG. 1. Relation betweeen effects on the cerebral cortex and dipole moments of $\omega$-aminoacids. Ordinate-excitatory $(+)$ and inhibitory $(-)$ power (arbitrary scale); Abscissa-carbon atom numbers between carboxyland amino-group. 

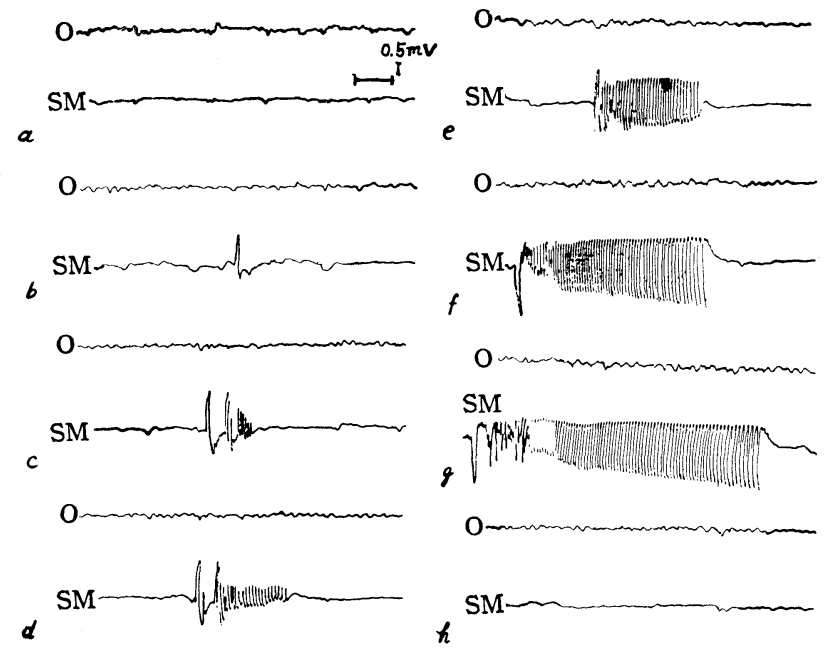

FIG. 2. Antagonism between the actions of $\varepsilon$-aminocaproic acid and $\gamma$-aminobutyric acid. Rabbit (2.4 kg) $a$-control, $b$-1 min. $40 \mathrm{sec}$. after the application of $6 \times 10^{-1} \mathrm{M} \mathrm{EACA}, c$ and $d$ respectively $1 \mathrm{~min} .20 \mathrm{sec}$. and $2 \mathrm{~min}$. after $8 \times 10^{-1} \mathrm{M} \mathrm{EACA}$, $\boldsymbol{e}-5 \mathrm{~min}$. after washing with fresh Ringer, $f, g$ and $h-1,2.5$ and $3 \mathrm{~min}$. after the application of $1 \times 10^{-1} \mathrm{M} \mathrm{GABA}$.

Chemicals were applied topically to the sensorimotor area. SM tracing was recorded on the sensorimotor area, and 0 tracing on the occipital area. If not otherwise described, upward deflexion is negative and horizontal bar represents 1 sec. in all the following figures.
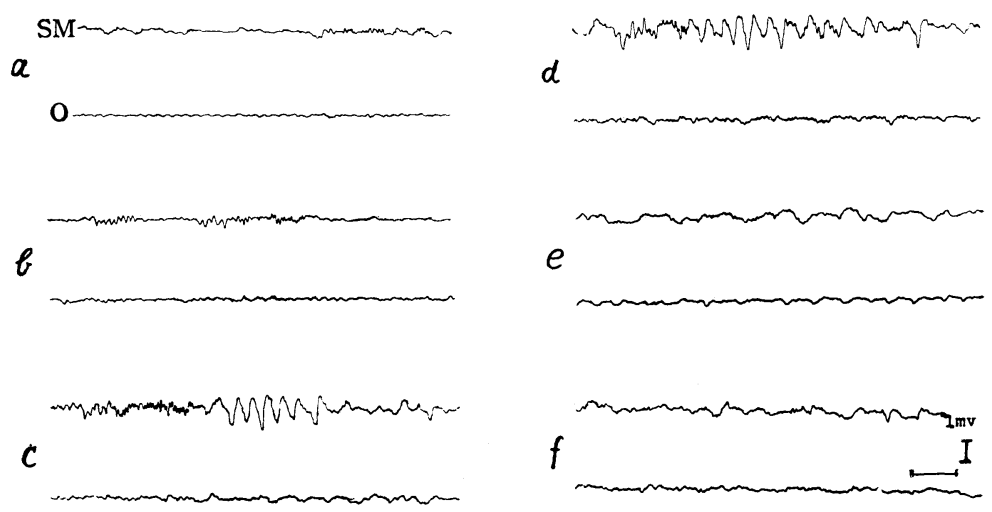

FIG. 3. Effects of $\alpha$-aminoisobutyric and $\beta$-aminoisobutyric acid on the spontaneous cortical activity of a rabbit $(2.3 \mathrm{~kg})$. $a$-control, $b, c$ and $d-4,19$, and $21 \mathrm{~min}$. after the application of $5 \times 10^{-1} \mathrm{M} \beta$-aminoisobutyric acid. $e-7 \mathrm{~min}$. after the cortex was washed with fresh Ringer. $f$ - $11 \mathrm{~min}$. after the application of $10 \times 10^{-1} \mathrm{M} \alpha$-aminoisobutyric acid. 
of $\omega$-aminoacids the distance between carboxyl- and amino-group may play an essential role.

\section{The inhibitory action of some choline derivatives}

The action of three choline derivatives on evoked potentials was investigated in rabbits and cats. Two of them were aminocholines, that is $\gamma$-aminobutyryl choline and glutamyl-choline, and the other was carbamyl choline.

\section{A. Effect on primary sensory potential}

All three cholines showed an inhibitory action on the primary sensory evoked potential recorded from the surface of the cat's posterior sigmoid gyrus in response to the stimulation of the contralateral peroneal nerve. $\gamma$-Aminobutyryl choline (GABA-Ch) showed the most powerful inhibitory action among them.

Carbamyl choline depressed considerably the primary evoked potentials at the concentration of $3 \times 10^{-2} \mathrm{~mol} / \mathrm{l}$. and glutamyl choline also at $4 \times 10^{-2} \mathrm{~mol} / 1$. (fig. 4). But, they did not show any appreciable effect on the primary sensory response at the concentration of $10^{-3} \mathrm{~mol} / 1$.

On the other hand, considerable suppression on the primary sensory response was observed at $2-4 \times 10^{-5} \mathrm{~mol} / 1$. of GABA-Ch. At the concentration of $4.0 \times 10^{-4} \mathrm{~mol} / 1$., GABA-Ch inhibited it remarkably (fig. 5).
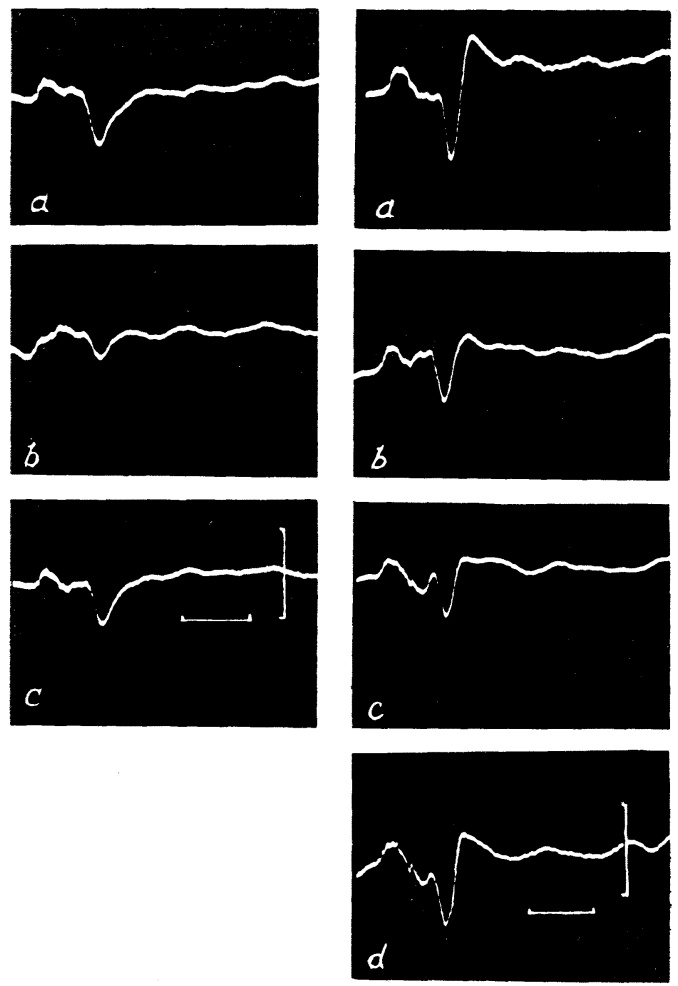

FIG. 4. Effects of glutamyl and carbamyl choline on the primary sensory potential evoked in the posterior sigmoid gyrus by stimulation of the contralateral peroneal nerve. Cat $(2.2 \mathrm{~kg})$ Left column: $\boldsymbol{a}$-control, $b$-effect of glutamyl choline $\left(4 \times 10^{-2}\right.$ $\mathrm{mol} / \mathrm{l}$.), observed in $2 \mathrm{~min}$. after application. $c$-recovery $2 \mathrm{~min}$. after washing off. Right column: $\boldsymbol{a}$-control, $b$ and $c-30 \mathrm{sec}$. and $4 \mathrm{~min}$. after the application of carbamyl choline $(3 \times$ $10^{-2} \mathrm{~mol} / \mathrm{l}$.). $d$-recovery after $1 \mathrm{~min}$. washing the cortex with fresh Ringer. The horizontal and vertical bar represent respectively $20 \mathrm{msec}$. and $0.5 \mathrm{mV}$. 

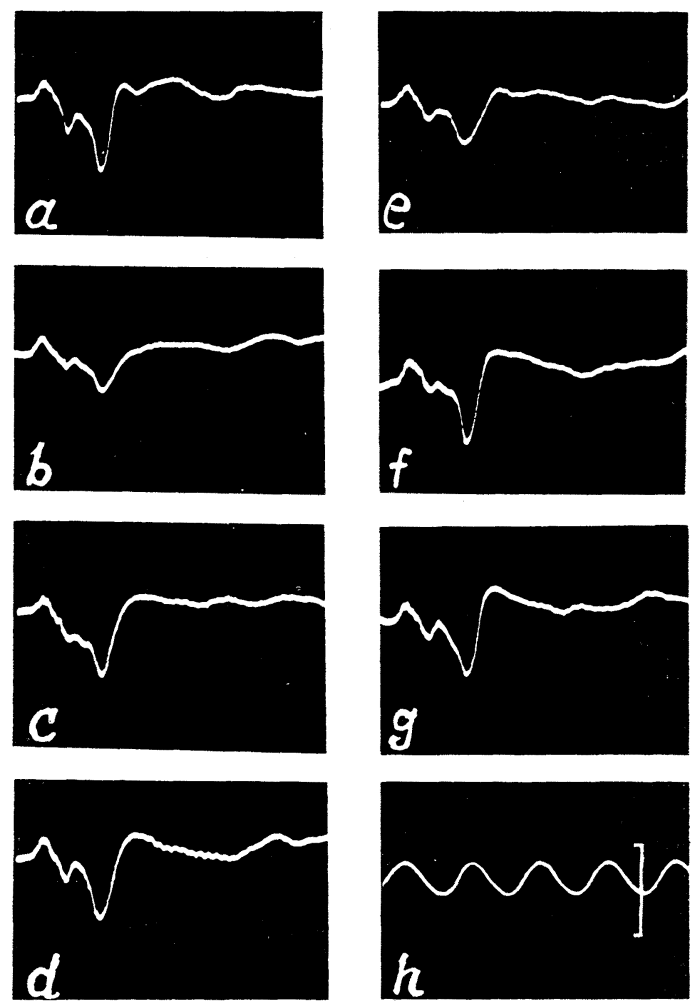

FIG. 5. Effect of GABA-Ch on the primary sensory evoked potential. Cat $(2.2 \mathrm{~kg})$. $a$-control, $b$-effect of $4.0 \times 10^{-4} \mathrm{M}$ GABA-Ch 30 sec. after application, $c$ and $d$-recovery $(30 \mathrm{sec}$. and $5 \mathrm{~min}$. respectively) after the cortex was washed with fresh Ringer, $e$-effect of $4.0 \times 10^{-5} \mathrm{M}$ GABA-Ch 3 min. after application, $f$ and $g$-recovery after 1 and 2 min., $h$-50 cps; The vertical bar represents $0.5 \mathrm{mV}$.

Both the positive and the negative waves in the primary sensory evoked potential were reduced in amplitude by the three cholines. Another effect of them was a prolongation of the positive wave. Washing the cholines from the cortex with fresh Ringer solution, rapid recovery of the original wave form occurred.

\section{B. Effect on direct cortical response}

They showed likewise the inhibitory action on the direct cortical response. The action of GABA-Ch was exceedingly more powerful than those of the others. It showed an appreciable inhibition in the concentrations of $2-4 \times 10^{-5}$ $\mathrm{mol} / \mathrm{l}$.

The example furnished in fig. 6 shows the effect of GABA-Ch on the cortical response to direct stronger stimulations in a rabbit under urethane anesthesia. It caused marked reduction in amplitudes of the initial and the 

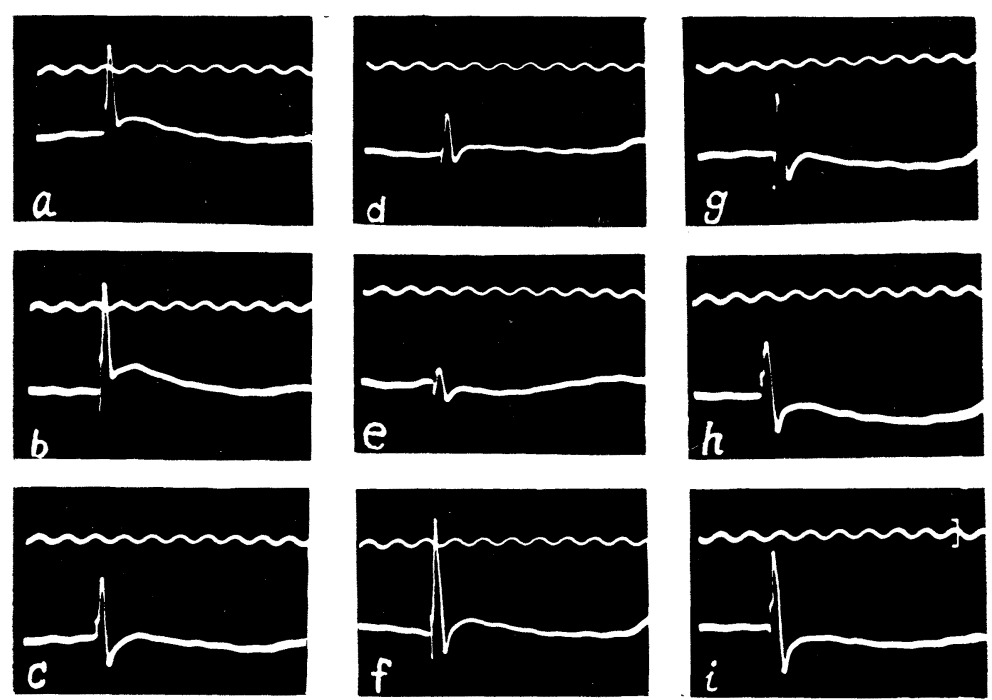

FIG. 6. Effect of $\gamma$-aminobutyryl choline on the dendritic potential at $2 \mathrm{~mm}$. distant from the stimulating electrodes in a rabbit $(2.0 \mathrm{~kg})$. $a, b$-control, $c, d$ and $e$-effect of $4.0 \times 10^{-3} \mathrm{M} \mathrm{GABA}-\mathrm{Ch} 3,4$ and $6 \mathrm{~min}$. after application, $f$-recovery $2 \mathrm{~min}$. after the cortex was washed with fresh Ringer, $g$-recovery after $16 \mathrm{~min}$., $h$-effect of $4.0 \times 10^{-4} \mathrm{M} \mathrm{GABA}-\mathrm{Ch}$ 5 min. after application, $i$-recovery $1 \mathrm{~min}$. after washing. Upper tracing-50 cps. The vertical bar represents $0.5 \mathrm{mV}$.

secondary surface negative waves, but left the positive component rather unaffected.

Washing the chemical from the cortex, rapid recovery was observed. At the beginning phase of the recovery, increase in amplitude of the initial negative wave beyond its original value was sometimes observed.

\section{Inhibitory actions of GABA, GABA-Ch and their related substances on metrazol-spike}

5-10\% metrazol, applied topically to the cortex, caused discharge of cortical elements repeatedly. Some substances were applied simultaneously with metrazol and their effects on metrazol spike were compared.

Although $\alpha$-aminoacids, such as glycine and $\alpha$-aminobutyric acid did not show any appreciable inhibitory or facilitatory effect on metrazol spikes, some $\omega$-aminoacids exhibited remarkable effect on it. $\beta$-Alanine caused complete inhibition on $10 \%$ metrazol excitation at the concentrarions of $1.3 \mathrm{~mol} / 1$.

In the case of GABA, the concentration necessary to inhibit completely the action of $10 \%$ metrazol was $1.5-4.0 \times 10^{-1} \mathrm{~mol} / \mathrm{l}$. (on the average of 5 examples $3.1 \times 10^{-1} \mathrm{M}$ ). In lower concentrations, GABA caused frequently reversal in polarity of metrazol spike. However, this reversal was not accompanied with suppression of convulsion or inhibition on spread of excitation to distant loci. 
Three choline derivatives likewise produced inhibition on metrazolexcitation. Among them, GABA-Ch showed the most powerful inhibitory effect. It caused complete inhibition on the excitatory action of $10 \%$ metrazol in the concentrations of $2-4 \times 10^{-4} \mathrm{~mol} / \mathrm{l}$. (on the average of 5 examples-3.0 $\times$ $\left.10^{-4} \mathrm{M}\right)$. Therefore, its inhibitory power was estimated at about 1000 times stronger as that of GABA.

The inhibitory actions of GABA and GABA.Ch were compared in the same preparations, because the excitability of the cortex considerably varied from preparation to preparation. Fig. 7 shows an example of such experiments. In this experiment, phase reversal in metrazol-spikes took place following the application of $1.0 \times 10^{-1} \mathrm{~mol} / \mathrm{l}$. of GABA. However, GABA-Ch did not invert the phase of metrazol-spikes.
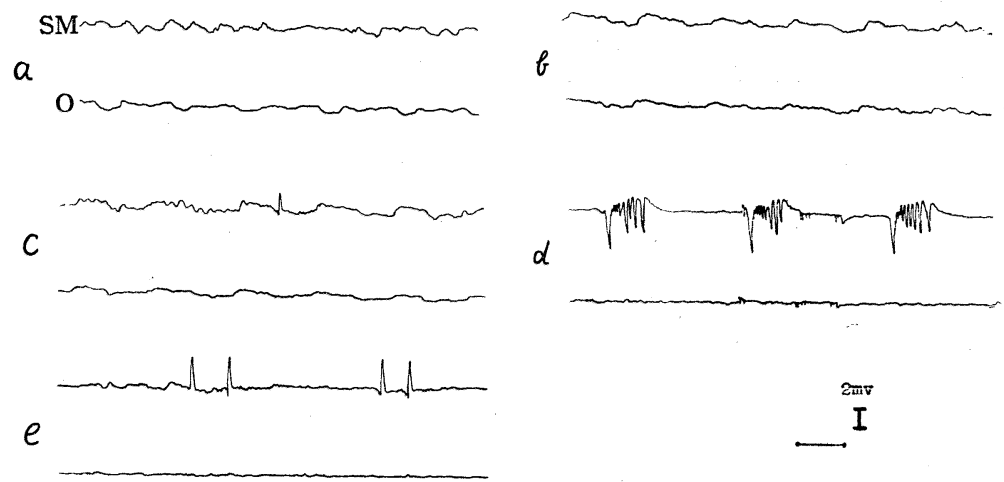

FIG. 7. Comparison of inhibitory action between $\gamma$-aminobutyric acid and $\gamma$-aminobutyryl choline. Rabbit $(2.3 \mathrm{~kg})$. At first, $10 \%$ metrazol and $3.8 \times 10^{-4}$ M GABA-Ch were topically applied to the sensorimotor area $(a)$, next, $10 \%$ metrazol and $3.0 \times 10^{-1} \mathrm{M} \mathrm{GABA}(b), 10 \%$ metrazol and $1.9 \times 10^{-4} \mathrm{M} \mathrm{GABA}-\mathrm{Ch}$ $(c), 10 \%$ metrazol and $1.0 \times 10^{-1} \mathrm{M} \mathrm{GABA}(d), 10 \%$ metrazol and $1.0 \times 10^{-4} \mathrm{M}$ GABA-Ch $(e)$ were respectively applied. Each application lasted for $5 \mathrm{~min}$. and then the chemical was washed off with fresh Ringer solution. After more than 5 min., next application was started. Metrazol-GABA-Ch spike was surface-negative, metrazol-GABA spike surface-positive.

Subsequently, the inhibitory powers of glutamyl choline, carbamyl choline and GABA were compared in the same preparations. Glutamyl choline inhibited completely the excitatory action of $5 \%$ metrazol at $2 \times 10^{-2} \mathrm{~mol} / \mathrm{l}$. Whereas, the concentrarions necessary for carbamyl choline and GABA to inhibit it completely were respectively $3 \times 10^{-2}$ and $1 \times 10^{-2} \mathrm{~mol} / 1$.

Among the isomers of GABA, $\alpha$-Amino- $n$-butyric acid and $\alpha$-aminoisobutyric acid did not show any appreciable inhibitory effect on $10 \%$ metrazolseizure, but $\beta$-aminoisobutyric acid had an inhibitory action similar to that of $\beta$-alanine.

The inhibitory action of GABA on convulsive seizures induced by intravenous injections of $25 \mathrm{mg}$. metrazol was compared with that of sodium phenobarbital. They were administered intravenously together with metrazol. 
About $5 \mathrm{mg}$. GABA could inhibit metrazol seizure completely, but $40 \mathrm{mg}$. of phenobarbital were necessary for complete suppression.

In some experiments, injections of metrazol were performed after full development of the effect of phenobarbital, taking the well-known progressive effect of barbiturates $(5,6,7)$ under consideration. In such instances, $35 \mathrm{mg}$. of phenobarbital could not completely inhibit seizures induced by $25 \mathrm{mg}$. of metrazol.

Intravenous injection of the same amount of GABA-Ch as GABA could not inhibit metrazol-seizure. This fact may suggest that GABA-Ch is promptly destructed in blood.

\section{DISCUSSION}

Some intimate relationship between the distances of amino- and carboxylgroup and the effects on neuronal functions of $\omega$-amino-acids was at first noticed by Takahashi et al. $(1,8)$. This interesting pnenomenon was also confirmed by Grundfest and his associates (2).

The significance of the dipole moments of $\omega$-aminoacids in their effects on the activity of the vasomotor center was essential (8). However, in the case of their effects on the cortical activity the relation was complicated; that is, shorter chain $\omega$-aminoacid series were inhibitory and longer chain series excitatory. For solution of this puzzle, the idea of competition seems not to be valid, because Grundfest et al. (2) showed that the excitatory action of $\mathrm{C}_{7}$ member was stronger than that of $\mathrm{C}_{6}$ member.

Another assumption seems to us more reasonable that the duality in the actions of these $\omega$-aminoacids is due to the difference of their sites of action. The question at issue in this postulate is the determination of the site of action of GABA.

In regard to this problem, attention of Grundfest has been focused on the phase-inverting action of GABA $(2,3,9)$. The inhibitory action of GABA was not so much powerful, comparing with that of choline derivatives. However, the phase inverting -action was characteristic of GABA. For example, GABA$\mathrm{Ch}$ that was recently synthetized by Kuriaki (10) showed more powerful innibitory effect on evoked potentials or metrazol spikes, but did not cause any phase reversal in the polarity of potentials.

As a consequence, the mechanism of phase reversal action must be analyzed, in order to elucidate the physiological role of GABA on the cortical activity. We will treat this problem in the following papers.

In a preliminary investigation, we observed that GABA, applied to cat's motor cortex induced convulsion in higher concentrations. However, it has been recognized that the phenomenon is due to some contamination contained in ill-prepared GABA.

\section{SUMMARY}

Pharmacological actions of various aminoacids and choline derivatives on the cortical activity of rabbits and cats were tested electrophysiologically. 
1. Among aminoacids, only $\omega$-aminoacids have been found to have pharmacological action on the cortex. Their shorter chain series showed inhibitory action. The inhibitory action of GABA was $c a .13$ times stronger as that of $\beta$-alanine. Whereas, $\varepsilon$-aminocaproic acid showed an excitatory action. The excitatory action of $10 \% \varepsilon$-amino-caproic acid was completely inhibited by $1 \%$ GABA.

2. GABA-Ch, glutamyl-choline and carbamyl-choline suppressed the primary sensory evoked potential and the direct cortical response. Among them, GABA-Ch showed the most powerful inhibition.

3. In the inhibition on metrazol spike, GABA-Ch was about 1000 time as active as GABA: glutamyl or carbamyl choline was about half or one-third as active.

4. By intravenous injection, the inhibitory action of GABA on seizures induced by metrazol was compared with that of sodium phenobarbital. Its inhibitory effect was prompt and much stronger than that of phenobarbital.

5 . Among isomers of GABA, only $\beta$-aminoisobutyric acid showed a slight inhibitory action.

6. GABA and $\beta$-aminoisobutyric acid could elicit surface positive potentials. The phase-inverting action of GABA on spikes induced by metrazol or $\varepsilon$-aminocaproic acid was remarkable.

GABA-Ch or other cholines did not show such effect.

7. A characteristic action of GABA on the electrical activity of the cortex seems to be phase-inverting.

We are thankful to Prof. Kuriaki for the gift of GABA.Ch and to Dr. Otsu for the gift of many pure aminoacids.

\section{REFERENCES}

1. TAKahashi, H. AND Y. Tsukada. J. Physiol. Soc. Jap. 14: 227, 1952 (in Japanese).

2. Purpura, D. P., M. Girado And H. Grundfest. Proc. Soc. Exptl. Biol. Med. 95: 791, 1957.

3. Purpura, D. P., M. Girado and H. Grundfest. Science 125: 1200, 1957.

4. IWAMA, K. AND H. H. JASPer. J. Physiol. 138: 365, 1957.

5. Hoagland, H., H. E. Himwich, E. Campbell, J. F. Fazekas and Z. Hadidian. $J$. Neurophysiol. 2: 276, 1939.

6. Cohn, R. And S. Katenelbogen. Proc. Soc. Exptl. Biol. Med. 49: 560, 1942.

7. Brazier, M. A. B. AND J. E. Finesinger. Arch. Neurol. Psychiat. 53: 51, 1946.

8. Takahashi, H., M. Tiba, M. IIno and T. Takayasu. Jap. J. Physiol. 5: 334, 1956.

9. Grundfest, H. Physiol. Revs. 37: 337, 1957.

10. KURIAKI, K. ET AL. Nature 181: 1336, 1958. 